\title{
Autonomous Operations in Optical Networks
}

\author{
Alba P. Vela*, Marc Ruiz and Luis Velasco \\ Universitat Politècnica de Catalunya, Barcelona, Spain \\ e-mail: apvela@ac.upc.edu
}

\begin{abstract}
Machine Learning (ML) has already proven its benefits for network operation, being a sub-domain of artificial intelligence, it is highly suitable for complex system representation. In this paper, basic ML concepts are reviewed, as well as its integration into existing network control and management planes. Then, a use case focused on soft-failure detection is presented in detail covering optical spectrum analysis and ML algorithms; the technique relies on the widespread deployment of cost-effective optical spectrum analyzer (OSA). Finally, the retrieved optical parameters are analyzed using ML algorithms giving rise to illustrative results.
\end{abstract}

Keywords: Machine Learning, Optical Networks

\section{INTRODUCTION}

Transport networks are complex interacting systems, involving cloud operations and core and metro transport. With localized and highly engineered operational tools, it is typical of these networks to take several weeks to months for any changes, upgrades or service deployments to take effect. Machine Learning (ML) is highly suitable for complex system representation as it enables this learning paradigm [1]. ML may be used to achieve network-domain goals including root-cause analysis and failure localization [2]-[4], as well as other related capital operational expenditure savings. ML algorithms comprise of a unique ability to learn system behavior from past data, and estimate future response based on the learned system model.

Supported by the recent improvements in computational hardware and parallel computing, the commercialization of big data storage, and processing frameworks, and the introduction of Software-defined networking (SDN) / Network Function Virtualization (NFV) platforms, several networking challenges may be partially or fully addressed using ML paradigms. We review several ML concepts and its integration into the control, orchestration and management (COM) system. Finally, we focus on fault management and cover degradation detection and localization, as early detection of equipment failure states and consequent remedial actions can prevent network downtime and enable scheduled preventive maintenance.

\section{OVERVIEW OF MACHINE LEARNING}

ML is typically thought of as a universal toolbox, ready to be used for classification, i.e., identifying to which of a set of categories a new observation belongs to, and regression, i.e., estimating the relationships among variables. In fact, it is a diverse field comprising of various constituents and includes data collection and transformation, model selection and optimization, performance evaluation, visualization, integration, etc. ML approaches may be categorized based on objectives of the learning task, where these objectives may target pattern identification for classification and prediction, learning for action, or inductive learning methods. The algorithms may be classified into three distinct learning families: i) supervised learning, ii) unsupervised learning and iii) reinforcement learning. Let us now introduce the ML families, as depicted in Fig. 1, together with some typical ML algorithms, and their respective applications in optical networking.

- Supervised Learning: Supervised learning makes use of known output feature(s), named labels, to derive a computational relationship between input and output data. An algorithm iteratively constructs a ML model by updating its weights, based on the mapping of a set of inputs to their corresponding output features. Examples of algorithms are Artificial Neural Networks (ANN), K-nearest Neighbors, and Support Vector Machine (SVM). Optical networking applications include resource optimization by estimation, and eventual prediction, of network state parameters for a given set of configurations (e.g., optimum launch power, etc.) Another application is ML-driven fault identification, based on historical traffic or network function patterns.

- Unsupervised Learning: While supervised learning provides a clean-slate approach to ML model construction, in practice, labeled data is neither easily accessible nor abundantly available. Unsupervised learning aims to build representation of a given data-set without any label-driven feedback mechanism. Examples of unsupervised learning algorithms are K-mean Clustering, Principal Component Analysis (PCA), and Self Organizing Maps (SOM). Unsupervised learning models may be naturally used for clustering of transport channels, nodes or devices, based on their temporal and spatial similarities. Applications include traffic migration, spectral slot identification, etc.

- Reinforcement Learning: Reinforcement learning refers to ML mechanisms without an explicit training phase. Reinforcement learning aims to build and update a ML model based on an agent's interaction with its own environment. The key difference with respect to supervised learning techniques is that labeled input- 
output features are not provided, but the relationship is rather learned via application of initial model to test data. Examples of algorithms include Q-learning, Random Forest, and Bayesian Network. One of their core applications in optical networks is network self-configuration, including resource allocation and service (re)configurations both for physical and virtual infrastructure.

\begin{tabular}{|c|c|c|c|}
\hline Supervised & Unsupervised & Semi-Supervised & Reinforcement \\
\hline $\begin{array}{l}\text { Input data is called training } \\
\text { data and has a known label. } \\
\text { Applications: } \\
\text { - Regression } \\
\text { - Classification }\end{array}$ & $\begin{array}{l}\text { Input data is not labeled. } \\
\text { Extracts general structures. } \\
\text { Applications: } \\
\text { - Clustering } \\
\text { - Association }\end{array}$ & $\begin{array}{l}\text { Input data is a mixture of } \\
\text { labeled and unlabeled data. } \\
\text { Applications: } \\
\text { - Clustering } \\
\text { - Classification }\end{array}$ & $\begin{array}{l}\text { Reward feedback is required } \\
\text { to learn its behavior. } \\
\text { Applications: } \\
\text { - Classification } \\
\text { - Control }\end{array}$ \\
\hline $\begin{array}{l}\text { - Artificial neural networks } \\
\text { - K-nearest neighbors } \\
\text { - SVM }\end{array}$ & $\begin{array}{l}\text { - K-means clustering } \\
\text { - } \mathrm{PCA} \\
\text { - Kohonen maps }\end{array}$ & $\begin{array}{l}\text { - } \text { Maximum likelihood } \\
\text { learning } \\
\text { - } \text { Generative models }\end{array}$ & $\begin{array}{ll}\text { - } & \text { Q-learning } \\
\text { - } & \text { Multi-armed bandits } \\
\text { - } & \text { MDP }\end{array}$ \\
\hline $\begin{array}{l}\text { - QoT estimation/prediction } \\
\text { - Predictive maintenance }\end{array}$ & $\begin{array}{ll}\text { - } & \text { Traffic clustering } \\
\text { - } & \text { Signal dimension reduction } \\
\end{array}$ & $\begin{array}{ll}\text { - } & \text { Resource allocation } \\
\text { - } & \text { Network behavior analysis }\end{array}$ & 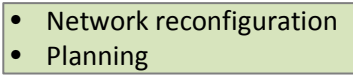 \\
\hline
\end{tabular}

Fig. 1. ML families.

\section{CONTROL, ORCHESTRATION AND MANAGEMENT}

To make use of advanced ML models, they need to be integrated into existing network software stack. However, multi-layer and multi-vendor network control and management is a complex task in itself, involving core and metro transport, as well as cloud operations. Although SDN has brought agility, flexibility, and scalability, into the network control as compared to traditional control and management platforms, enabling centralized, programmable, and automated services across multiple domains, to attain true network automation, centralized SDN control needs to be augmented with instantaneous data-driven decision-making using advanced monitoring and ML tools, feeding management and control plane alike. The discussions around SDN have mostly focused on separation of data and control planes, with little attention on operational feedback loop, including monitoring, intelligence and management functionalities. Fig. 2 captures this theme and presents a high-level network architecture, where central offices (CO) consist of intra- and inter- data center (DC) infrastructure. The intra-DC resources comprise storage, compute and network, whereas inter-DC connectivity is provided by a transport network. Resources are continuously monitored, exposing real-time network states to the analytics stage, which in turn feeds the COM system [5].

This holistic platform not only caters for centralized and programmable control, but also makes ML-driven decisions to trigger actions, essentially connecting data-driven automation with policy-based orchestration and management. To this end, the COM architecture includes the NFV Orchestrator providing network services, the virtual infrastructure manager (VIM) coordinating and automating DC workflows, the network orchestrator adopting hierarchical control architectures with a parent SDN controller abstracting the underlying complexity, and a monitoring and data analytics (MDA) controller that collates monitoring data from network, cloud and applications and contains ML algorithms.

Regarding MDA, a hybrid architecture may be

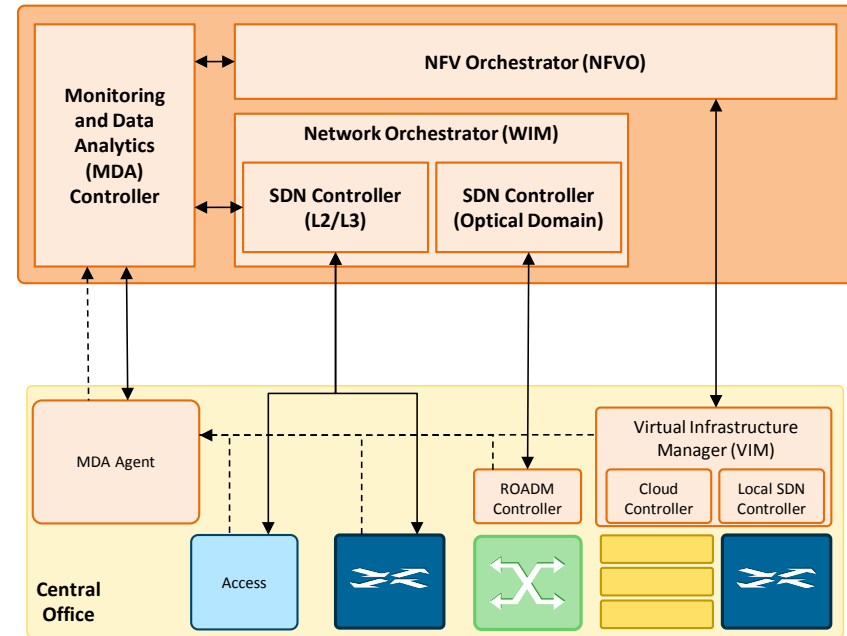

Fig. 2. Autonomic networking architecture. envisioned, where every CO includes a distributed MDA agent that collates monitoring data from the network, cloud and applications; and a centralized MDA controller [6]. The MDA agent exposes two interfaces toward the MDA controller for collecting monitoring and telemetry data. In addition, specific interfaces for monitoring control allow the MDA agent to connect with the network nodes. The MDA agent includes a local module containing data analytics applications for handling and processing data records. The data analytics capabilities deployed close to the network nodes enable local control loops, i.e. localized data analysis, and consequent updated configurations.

The centralized MDA controller abstracts monitored data via suitable interfaces and implements a ML-based learning engine, where ML algorithms analyze monitoring data to discover patterns. Such knowledge can be used to make predictions, detect anomalies before they negatively impact the network. Such events can be 
notified in advance to the corresponding COM module (SDN controller or orchestrator), together with a recommended action. Note that a recommended action is a suggestion that the COM module can follow or just ignore and apply its own policies. The notification might trigger a network re-configuration, hence closing the loop and adapting the network to the new conditions. It is worth highlighting the importance of the control loops for network automation, as it fundamentally changes the way networks are operated today -empowering truly dynamic and autonomous operation. As examples of control loops, let analyze that of soft-failure detection.

\section{SOFT-FAILURE DETECTION}

Let us now introduce an application of ML related to fault management in optical networks. Many commercial equipment tolerates some errors until automatically tearing down the connection when some system thresholds are exceeded. While a restoration procedure could be initiated to recover the affected traffic, it would be desirable to anticipate such degradations and localize the root-cause of the (soft) failure so the lightpath can be re-routed before it is disrupted; note that failure localization is required to exclude the failed resources from path computation, as well as to schedule maintenance tasks. In addition, proactive failure detection would also allow time to plan the re-routing procedure, e.g., during off-peak hours.

Soft-failures can degrade lightpaths' quality of transmission and introduce errors in the optical layer that might impact on the quality of the services deployed on top of such networks. Some soft-failures affect the shape of optical signals and they can be detected by the MDA agents at intermediated nodes analyzing the optical spectrum acquired by local Optical Spectrum Analyzers (OSA). Note that the acquired optical spectra entails large amount of data (e.g., 6,400 frequency-power $(<f, p>)$ pairs for the C-band for OSAs with $625 \mathrm{MHz}$ resolution), so local analysis carried out at the MDA agents greatly reduces the amount of data to be conveyed to the MDA controller. Upon detection of a soft-failure, the MDA agent notifies the MDA controller, which is able to correlate notifications received from several MDA agents and for several lightpaths to localize the element that is causing the failure.

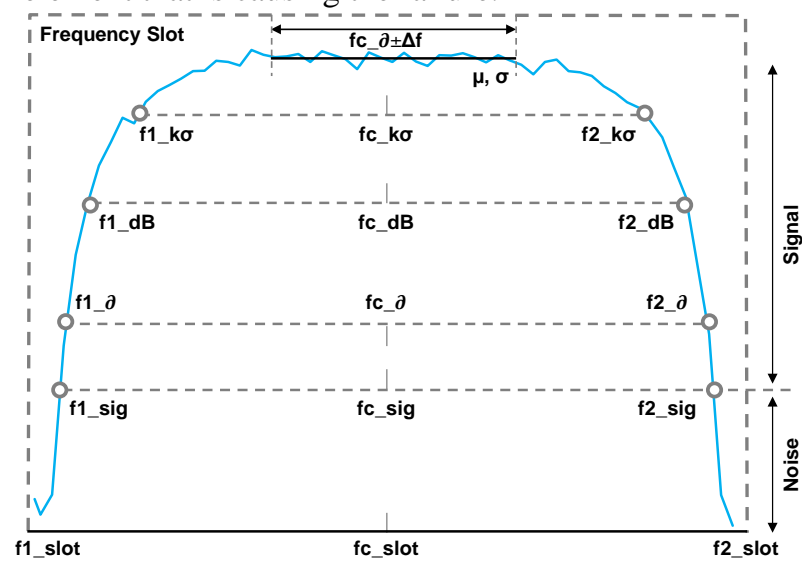

Fig. 3 shows an example of the optical spectrum of a 100Gb/s DP-QPSK modulated signal. By inspection, we can observe that a signal is properly configured when: $i)$ its central frequency $\left(f_{c}\right)$ is around the center of the allocated slot; ii) its spectrum is symmetrical 票 with respect to $f_{c}$; and iii) the effect of filter cascading is limited to a value given by the number of filters that the signal has traversed. However, when a filter failure occurs, the spectrum is distorted, and the distortion can fall into two categories: $i$ ) the optical spectrum is asymmetrical as a result of one or more filters are misaligned with respect to the $f_{c}$ of the slot allocated for the signal (filter shift, FS), and ii) the edges of the optical spectrum look excessively rounded for the

Fig. 3. Example of optical spectrum and signal features. number of filters, because of the bandwidth of a filter is narrower than the frequency slot width allocated for the signal (filter tightening, FT). Classifiers, based on e.g., SVMs can be used to detect such filter failures in intermediate nodes, so the optical node responsible for the failure can be determined. Once localized, the SDN controller can re-route the affected lightpath excluding the failed resource.

To detect the above distortions, an optical spectrum (represented by an ordered list of $\langle f, p>$ pairs) can be processed to compute relevant signal points that facilitate its diagnosis. Before processing an optical spectrum, it is normalized to $0 \mathrm{dBm}$. Next, signal features are computed as follows [2]: i) equalized noise level, denoted as sig (e.g., -60dB + equalization level); ii) edges of the signal, computed using the derivative of the power with respect to the frequency, denoted as $\partial$; iii) statistics $\mu$ and $\sigma$ of the central part of the signal, computed using the edges from the derivative $\left(f_{c_{\_} \partial} \pm \Delta f\right)$; iv) family of power levels computed with respect to $\mu-k \sigma$, denoted as $k \sigma$; and $v$ ) a family of power levels computed with respect to $\mu$ minus $k \mathrm{~dB}$, denoted as $d B$. Using these levels, two cut-off points can be generated and denoted as $f_{1(\cdot)}$ and $f_{2(\cdot)}$ (e.g., $\left.f_{1 s i g}, f_{l o}, f_{1 \mathrm{~dB}}, f_{1 \mathrm{k} \sigma}\right)$. Besides, the assigned slot is denoted as $f_{1 s l o t}, f_{2 s l o t}$. Other features are also computed as linear combinations of the above ones.

These features are used as input for the subsequent failure detection and identification modules. Although relevant metrics are computed from an equalized signal, signal distortions due to filter cascading effect has not been corrected yet. As mentioned above, this effect might result in an incorrect diagnosis of a potential filter problem. To overcome this, we apply a correction mask to the measured signal. Such correction masks can be easily obtained by means of theoretical signal filtering effects or experimental measurements.

The two considered filter failures scenarios are illustrated in Fig. 4, where the solid line represents the optical spectrum of the normal signal expected at the measurement point and the solid area represents the optical 


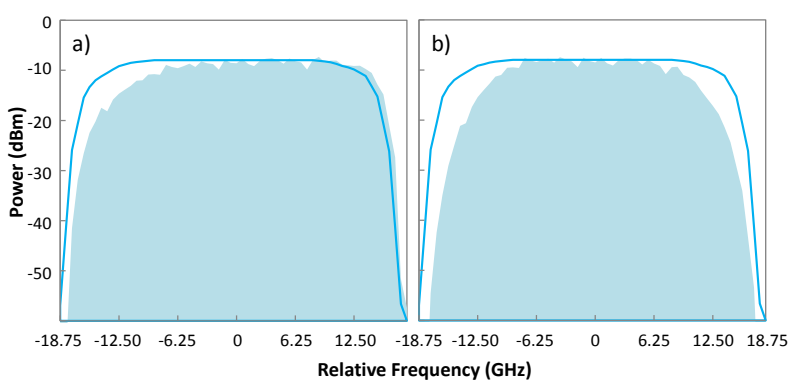

Fig. 4. Example of filter failures: FS (a) and FT (b).

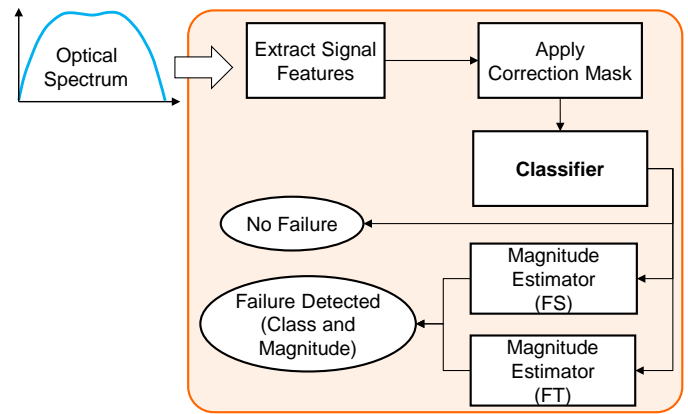

Fig. 5. Workflow for failure detection and identification

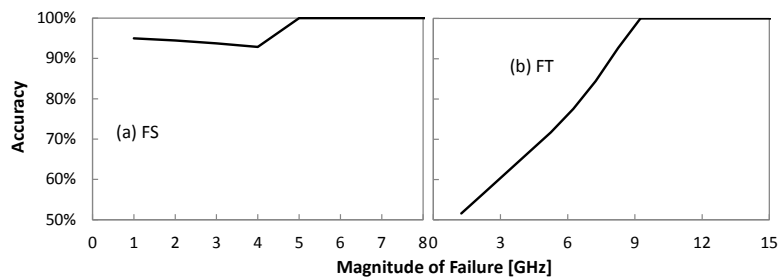

Fig. 6. Accuracy for failure identification spectrum of the signal with failure. In case of filter shift, a $10 \mathrm{GHz}$ shift to the right was applied (Fig. $4 \mathrm{a}$ ), whereas the signal is affected by a $20 \mathrm{GHz}$ FT in Fig. 4b.

We make use of classification and regression algorithms. In the case of classification, the objective is to classify unknown received data, e.g., an optical signal, and decide whether the signal belongs to the normal class, the FS class, or the FT class, whereas regression is used to estimate the magnitude of a failure. Once the optical spectrum of a signal has been acquired, and processed as described above, failure analysis is carried out. Fig. 5 summarizes the workflow that returns the detected class of the failure (if any) and its magnitude. While ML algorithms are suitable for this task, we selected SVM for classification, and linear regression for prediction.

Let us know numerically study the proposed workflow using a testbed modeled in VPI Photonics, where the optical spectrum database was generated for training and testing the proposed algorithms. In this study, we focus on the cases where failure happens just at the 1st node. A large database of failure scenarios with different magnitude (1 to 15 $\mathrm{GHz}$ for FT and 1 to $8 \mathrm{GHz}$ for FS, both with 0.25 $\mathrm{GHz}$ step-size) was collected. Fig. 6a-b show the accuracy of identifying FS and FT, respectively, in terms of the failure magnitude. Every point in Fig. 6a-b is obtained by considering all the observations belonging to that particular failure magnitude, and above. This representation reveals the true accuracy of the classifier while considering failures with magnitude above certain thresholds. For instance, the accuracy of detecting FS in a dataset comprising observations larger than $1 \mathrm{GHz}$ (in our case it comprises of failures up to $8 \mathrm{GHz}$ in which there are equal number of observations per each magnitude) is around $96 \%$. On the other hand, the accuracy of the classifier becomes $100 \%$ for failures larger than $5 \mathrm{GHz}$.

Once the failures are detected, FS estimator (FSE) and FT estimator (FTE) can be launched to return the magnitude of the failures. In our case, the estimators predicted the magnitude of failures with very high accuracy, with mean square error (MSE) equal to 0.091 and 0.006 for FSE and FTE, respectively.

\section{CONCLUSIONS}

Traditional networks suffer from largely static operational and optimization practices that limit their scalability and efficiency. ML provides a collection of techniques to fundamentally adapt to the dynamic network behavior. While the application of ML for optical networks is still in its infancy; these learning-based techniques provide a promising platform for end-to-end network automation, including fault management.

\section{ACKNOWLEDGEMENTS}

The research leading to these results has received funding from the Spanish MINECO TWINS project (TEC2017-90097-R), from the EC through the METRO-HAUL project (G.A. no 761727), and from the Catalan Institution for Research and Advanced Studies (ICREA).

\section{REFERENCES}

[1] D. Rafique and L. Velasco, "Machine Learning for Optical Network Automation: Overview, Architecture and Applications,” IEEE/OSA JOCN, vol. 10, pp. D126-D143, 2018.

[2] A. P. Vela et al., "Soft failure localization during commissioning testing and lightpath operation," IEEE/OSA JOCN, vol. 10, pp. A27-A36, 2018.

[3] A. P. Vela et al., "BER Degradation Detection and Failure Identification in Elastic Optical Networks," IEEE/OSA Journal of Lightwave Technology (JLT), vol. 35, pp. 4595-4604, 2017.

[4] B. Shariati et al., "Learning from the Optical Spectrum: Failure Detection and Identification [Invited]," IEEE/OSA JLT, vol. 37, pp. 433-440, 2019.

[5] Ll. Gifre et al., “Autonomic Disaggregated Multilayer Networking,” IEEE/OSA JOCN, vol. 10, pp. 482-492, 2018.

[6] Ll. Gifre, J.-L. Izquierdo-Zaragoza, M. Ruiz, and L. Velasco, “Autonomic Disaggregated Multilayer Networking,” in IEEE/OSA JOCN, vol. 10, pp. 482-492, 2018. 